\section{(C) OPEN ACCESS}

\title{
Trimethoprim-sulfamethoxazole prophylaxis prevents severe/life-threatening infections following rituximab in antineutrophil cytoplasm antibody-associated vasculitis
}

\author{
Andreas Kronbichler, ${ }^{1,2}$ Julia Kerschbaum, ${ }^{2}$ Seerapani Gopaluni, ${ }^{1}$ Joanna Tieu, ${ }^{1}$ \\ Federico Alberici, ${ }^{1,3}$ Rachel Bronwen Jones, ${ }^{1}$ Rona M Smith, ${ }^{1}$ David R W Jayne ${ }^{1,4}$
}

\section{Handling editor Josef $\mathrm{S}$ Smolen \\ - Additional material is published online only. To view please visit the journal online (http://dx.doi.org/10.1136/ annrheumdis-2017-212861) \\ ${ }^{1}$ Vasculitis and Lupus Clinic, Addenbrooke's Hospital, Cambridge, UK \\ ${ }^{2}$ Department of Internal Medicine IV (Nephrology and Hypertension), Anichstraße, Innsbruck, Austria \\ ${ }^{3}$ Renal Medicine and Vasculitis Clinic, San Carlo Borromeo Hospital, Milan, Italy ${ }^{4}$ Department of Medicine, University of Cambridge, Cambridge, UK}

Correspondence to Dr Andreas Kronbichler, Vasculitis and Lupus Clinic, Addenbrooke's Hospital, Cambridge CB2 0QQ, UK; andreas.kronbichler@i-med. ac.at

Parts of this work were presented during the 52nd ERA EDTA Congress 2015 (London, UK) and during the ASN Kidney Week 2016 (Chicago, USA).

Received 16 December 2017 Accepted 11 June 2018

Published Online First 27 June 2018

\section{ABSTRACT}

Objective We aimed to assess risk factors for the development of severe infection in patients with antineutrophil cytoplasm antibody-associated vasculitis (AAV) receiving rituximab.

Methods 192 patients with AAV were identified. Univariate and multivariate analyses were performed to identify risk factors for severe infection following rituximab. Severe infections were classified as grade $\geq 3$ as proposed by the Common Terminology Criteria for Adverse Events V.4.0.

Results 95 severe infections were recorded in 49 (25.52\%) patients, corresponding to an event rate of 26.06 per 100 person-years. The prophylactic use of trimethoprim-sulfamethoxazole was associated with a lower frequency of severe infections ( $\mathrm{HR} 0.30,95 \% \mathrm{Cl}$ 0.13 to 0.69 ), while older age (HR $1.03,95 \% \mathrm{Cl} 1.01$ to 1.05 ), endobronchial involvement (HR $2.21,95 \% \mathrm{Cl}$ 1.14 to 4.26$)$, presence of chronic obstructive pulmonary disease (HR 6.30, 95\% Cl 1.08 to 36.75) and previous alemtuzumab use (HR 3.97, 95\% Cl 1.50 to 10.54) increased the risk. When analysis was restricted to respiratory tract infections (66.3\% of all infections), endobronchial involvement (HR 4.27, 95\% Cl 1.81 to 10.06), severe bronchiectasis (HR $6.14,95 \% \mathrm{Cl} 1.18$ to 31.91), higher neutrophil count (HR 1.19, 95\% Cl 1.06 to 1.33 ) and major relapse (HR $3.07,95 \% \mathrm{Cl} 1.30$ to 7.23) as indication for rituximab use conferred a higher risk, while refractory disease (HR $0.25,95 \% \mathrm{Cl} 0.07$ to 0.90 ) as indication had a lower frequency of severe infections.

Conclusions We found severe infections in one quarter of patients with AAV receiving rituximab. Trimethoprimsulfamethoxazole prophylaxis reduced the risk, while especially bronchiectasis and endobronchial involvement are risk factors for severe respiratory infections.

\section{INTRODUCTION}

Antineutrophil cytoplasm antibody (ANCA)-associated vasculitis (AAV) encompasses three entities, namely granulomatosis with polyangiitis (GPA, previously Wegener's granulomatosis), microscopic polyangiitis (MPA) and eosinophilic granulomatosis with polyangiitis (EGPA, previously Churg-Strauss Syndrome). The availability of ANCA facilitates diagnosis and treatment strategies, and has led to a better prognosis over recent decades. ${ }^{1}$ Nevertheless, comorbidities attributable to the persistence of the disease or side effects of treatment remain a challenge. Forty-eight per cent of deaths occurring during the first year are caused by infections and remain a major cause of mortality thereafter. ${ }^{2}$ Infectious complications have been studied especially in cyclophosphamide-treated patients. Several risk factors have been identified, including treatment intensity (cumulative steroid and cyclophosphamide dose), reduced creatinine clearance (estimated glomerular filtration rate (eGFR) of $\leq 30 \mathrm{~mL} / \mathrm{min}$ ) or dialysis dependency, older age and pulmonary involvement. ${ }^{3}$ Rituximab showed similar efficacy compared with a cyclophosphamide-based treatment in the induction of remission in two randomised controlled trials. However, rituximab did not show a reduced rate of severe infections compared with cyclophosphamide. ${ }^{4} 5$ Patients recruited into trials may have a lower adverse event rate due to rigorous monitoring and selection of patients according to exclusion criteria, ${ }^{6}$ and the rate of side effects might be even higher in routine practice. Several observational studies have reported severe/life-threatening infectious complications following rituximab, including cases with Pneumocystis jirovecii, Pseudomonas aeruginosa, pulmonary aspergillosis and progressive multifocal leukoencephalopathy. ${ }^{7-9}$ While $P$. jirovecii prophylaxis is widely accepted in patients receiving cyclophosphamide (CYC), no such recommendations exist for patients receiving rituximab.

This study investigated the frequency of severe/ life-threatening infections in 192 patients with AAV treated with rituximab. It also aimed to identify risk factors for severe infection in this patient population.

\section{METHODS}

\section{Study population}

This study included patients with AAV older than 18 years who were referred for rituximab to two tertiary care specialist centres, Addenbrooke's Hospital (Cambridge, UK) and the Medical University Innsbruck (Innsbruck, Austria), between 2004 and 2014. Diagnosis of AAV was established according to the European Medicines Agency (EMA) algorithm. ${ }^{10}$ Follow-up of patients began at the time of rituximab administration and ended on the date of death, the date patients were lost to follow-up, 2 years after first rituximab 
administration or on 1 January 2015, whichever occurred first. This study was conducted in accordance with the ethical principles stated in the Declaration of Helsinki. The Institutional Review Board of both university hospitals approved the use of anonymised patient data for research purposes.

\section{Clinical data}

The following data were obtained from the respective electronic medical records of the patients: demography (age, gender), diagnosis, date of diagnosis, time to rituximab, ANCA serotype, disease phenotype, organ involvement, prior immunosuppressive therapies, cumulative cyclophosphamide exposure (in grams), immunosuppression during the year before rituximab, concomitant treatment, laboratory values (serum creatinine, C reactive protein (CRP), erythrocyte sedimentation rate (ESR), neutrophils, white blood count (WBC), lymphocytes, CD3/CD4/CD8/CD19/CD56 counts, immunoglobulins), indication for the use of rituximab (see online supplementary appendix), comorbidities (including chronic obstructive pulmonary disease, diabetes mellitus, hypertension, chronic heart failure), smoking history, antibiotic prophylaxis (trimethoprim-sulfamethoxazole or others) and the occurrence of severe/life-threatening infections (grade $\geq 3$ ), as classified by the Common Terminology Criteria for Adverse Events (CTCAE) V.4.0 (see online supplementary appendix). ${ }^{11}$ Hypogammaglobulinaemia was defined as a IgG level of below $7 \mathrm{~g} / \mathrm{L}$. Patients with incomplete or missing medical records were excluded from further analyses. The cumulative doses of rituximab during follow-up were determined.

\section{Statistical analysis}

Categorical variables were compared using the $\chi^{2}$ test (or Fisher's exact test, when appropriate), and metric variables were compared using the Mann-Whitney U test. Metric variables are shown as median (and minimum to maximum), and nominal variables are shown as per cent (\%). Both univariate and multivariate Cox regression analyses were performed to determine significant risk factors for severe/life-threatening and respiratory infections. The occurrence of at least one episode of severe/ life-threatening infection during the follow-up period of 24 months was the outcome of interest. Kaplan-Meier plots and log-rank test were performed to assess univariate associations. All variables showing significant association with the dependent variable in the univariate Cox regression analysis were entered into a multivariate Cox regression model. A backward selection procedure was then used (with $\mathrm{p}$ values greater than 0.100 as the removal criterion, using Wald's test). Neutrophils correlated with WBC, CRP and ESR and sinusitis correlated with ear, nose and throat (ENT) involvement, thus only neutrophils at baseline and sinusitis were included in multivariate analysis. Results are expressed as HRs with 95\% CIs. All statistical analyses were performed with SPSS Statistics V.21.0 (IBM).

\section{RESULTS}

\section{Patient characteristics}

The total number of patients included in the analysis was 192 (134 with GPA, 28 with MPA and 30 with EGPA). Mean duration of initial diagnosis to initiation of rituximab was 4.33 years. Patients were followed for a mean time of 22.67 months from the time of rituximab initiation (mean rituximab dose $4.75 \mathrm{~g}$ ). Fortynine patients presented with 95 infectious complications classified as CTCAE V.4.0 $\geq 3$. In detail, 71 episodes were CTCAE V.4.0 grade 3, 23 as grade 4 and 1 as grade 5 (multiorgan failure as a consequence of sepsis related to an urinary tract infection). The overall event rate was 26.06 per 100 person-years. Twenty-five per cent of the observed infections occurred during the first 4 months of follow-up, while $50 \%$ and $80 \%$ were observed after 12 and 18 months, respectively. Antibiotic prophylaxis with trimethoprim-sulfamethoxazole was administered in 73 out of $192(38.02 \%)$. During the follow-up period, seven fatalities were recorded. Baseline characteristics of patients with severe infections and those without are depicted in table 1 .

\section{Infections}

Respiratory tract infection was the most common infectious complication $(n=63)$, followed by urinary tract $(n=12)$, gastrointestinal tract $(\mathrm{n}=8)$, mastoiditis/otitis externa $(\mathrm{n}=4)$, skin $(\mathrm{n}=3)$, sepsis/septicaemia with unidentified site of infection $(n=1)$, catheter-associated exit site infections $(n=1)$, orbital mass infection $(n=1)$, lacrimal gland abscess $(n=1)$ and eye $(n=1)$ (online supplementary table S1). Moreover, in cases with a positive microbial result, opportunistic pathogens were seen, including P. aeruginosa $(\mathrm{n}=4)$, Staphylococcus aureus including methicillin-resistant strains $(\mathrm{n}=4)$, Escherichia coli $(\mathrm{n}=3)$, Clostridium difficile $(\mathrm{n}=2)$, P. jirovecii $(\mathrm{n}=1)$, Legionella pneumophila $(\mathrm{n}=1)$ and invasive aspergillosis $(\mathrm{n}=1)$. In addition, one case of Campylobacter jejuni gastroenteritis was observed (online supplementary table S1 and online supplementary table S2).

\section{Rituximab treatment and risk of infections}

To identify specific risk factors associated with the development of infectious complications, univariate analysis was performed. Older patients (HR 1.02, 95\% CI 1.00 to 1.04), patients with endobronchial involvement (HR 2.44, 95\% CI 1.38 to 4.32) and severe bronchiectasis (HR 4.79, 95\% CI 1.47 to 15.59) were at increased risk for severe infections. Patients presenting with sinusitis (HR $0.48,95 \% \mathrm{CI} 0.27$ to 0.84 ) or in general ENT involvement (HR 0.46, 95\% CI 0.26 to 0.82 ) had fewer severe infections. While there was no correlation with serum creatinine, higher eGFR (HR 0.99, 95\% CI 0.98 to 1.00 ) emerged as a protective factor. Higher ESR (HR 1.11, 95\% CI 1.03 to 1.20), WBC (HR 1.06, 95\%CI 1.01 to 1.10), higher steroid doses (HR 1.02, 95\% CI 1.01 to 1.04) and an IgG decline $\geq 30 \%$ (HR 1.88, 95\% CI 1.04 to 3.39) at baseline were predictors of severe infections. Concomitant comorbidities, such as chronic obstructive pulmonary disease (COPD, HR 16.07, 95\% CI 4.41 to 58.49 ), diabetes (HR 2.35, 95\% CI 1.14 to 4.85 ) and reduced left ventricular ejection fraction/previous myocardial infarction (HR 2.21, 95\% CI 1.07 to 4.56) emerged as risk factors. Treatment with alemtuzumab (ALM) ever before rituximab was associated with an increased risk (HR 2.49, 95\% CI 1.05 to 5.91). Antibiotic prophylaxis to prevent $P$. jirovecii infections with trimethoprim-sulfamethoxazole reduced the risk of severe infections ( $\mathrm{HR} 0.45,95 \% \mathrm{CI} 0.23$ to 0.88). A multivariate logistic regression analysis revealed that the use of trimethoprim-sulfamethoxazole as prophylactic antibiotic measure had an impact on reduction of severe infections (HR $0.30,95 \% \mathrm{CI} 0.13$ to 0.69 ). Moreover, the use of trimethoprim-sulfamethoxazole significantly reduced the time to first significant infection $(\mathrm{p}=0.016)$ (table 2 and figure 1). Moreover, older age (HR 1.03, 95\% CI 1.01 to 1.05), endobronchial involvement ( $\mathrm{HR} 2.21,95 \% \mathrm{CI}$ 1.15 to 4.26 ), COPD (HR 6.30, 95\% CI 1.08 to 36.75) and ALM treatment before rituximab (HR 3.97, 95\% CI 1.50 to 10.54) emerged as independent risk factors to develop severe infections following rituximab (table 2). 


\section{Clinical and epidemiological research}

Table 1 Baseline characteristics of patients having severe infections versus those without severe infections

\begin{tabular}{|c|c|c|c|}
\hline & No severe infection $(n=143)$ & Severe infection $(n=49)$ & $P$ values \\
\hline \multicolumn{4}{|l|}{ Demographics } \\
\hline Age (years) & $56(16-85)$ & $60(22-82)$ & 0.023 \\
\hline Gender (male, \%) & 45 & 41 & 0.573 \\
\hline Type of vasculitis (\%) & & & 0.407 \\
\hline GPA & 71 & 65 & \\
\hline MPA & 13 & 20 & \\
\hline EGPA & 16 & 14 & \\
\hline \multicolumn{4}{|l|}{ Symptoms (\%) } \\
\hline B-symptoms (night sweat, fever, unintentional weight loss) & 21 & 14 & 0.353 \\
\hline Neuropathy & 27 & 24 & 0.774 \\
\hline Sinusitis & 72 & 53 & 0.015 \\
\hline Deafness/mastoiditis/otitis media & 31 & 22 & 0.266 \\
\hline Arthralgia & 45 & 33 & 0.117 \\
\hline \multicolumn{4}{|l|}{ Organ involvement (\%) } \\
\hline CNS & 7 & 7 & 1 \\
\hline Subglottic/tracheal stenosis & 12 & 14 & 0.661 \\
\hline Skin & 18 & 14 & 0.533 \\
\hline Kidney & 44 & 51 & 0.398 \\
\hline Eye & 29 & 17 & 0.112 \\
\hline Others & 7 & 8 & 1 \\
\hline ENT & 79 & 61 & 0.014 \\
\hline Lung & 54 & 65 & 0.162 \\
\hline \multicolumn{4}{|l|}{ Imaging findings (\%) } \\
\hline Pulmonary cavities & 24 & 26 & 0.75 \\
\hline Endobronchial & 20 & 41 & 0.004 \\
\hline Severe bronchiectasis & 1 & 8 & 0.054 \\
\hline \multicolumn{4}{|l|}{ Disease activity measures } \\
\hline BVAS & $6(0-28)$ & $6(0-18)$ & 0.602 \\
\hline DEI & $6(2-12)$ & $6(2-10)$ & 0.848 \\
\hline \multicolumn{4}{|l|}{ Laboratory values } \\
\hline Creatinine $(\mu \mathrm{mol} / \mathrm{L})$ & $86(45-1451)$ & $98(49-879)$ & 0.027 \\
\hline eGFR (MDRD/Modification of Diet in Renal Disease equation) $\mathrm{mL} / \mathrm{min} / 1.73 \mathrm{~m}^{2}$ & $75(3-163)$ & $60(5-155)$ & 0.002 \\
\hline CRP $(0-6 \mathrm{mg} / \mathrm{L})$ & $5.0(0.7-215.0)$ & $14.0(1.0-215.0)$ & 0.001 \\
\hline ESR (5-15 in the 1st hour) & $16(2-116)$ & $22(1-109)$ & 0.006 \\
\hline Neutrophils $\left(2-8 \times 10^{9} / L\right)$ & $7.1(2.0-18.6)$ & $8.3(2.4-21.4)$ & 0.025 \\
\hline WBC $\left(4-11 \times 10^{9} / L\right)$ & $9.4(3.6-42.0)$ & $10.7(3.3-24.4)$ & 0.006 \\
\hline Lymphocytes (1-4.5×109/L) & $1.0(0.1-3.7)$ & $1.0(0.4-4.5)$ & 0.145 \\
\hline CD19 (0.1-0.5) & $0.04(0.00-0.80)$ & $0.03(0.00-0.77)$ & 0.781 \\
\hline CD3 $(0.7-2.1)$ & $0.82(0.05-7.20)$ & $0.70(0.21-3.32)$ & 0.246 \\
\hline CD4 (0.3-1.4) & $0.48(0.03-1.98)$ & $0.38(0.11-2.80)$ & 0.303 \\
\hline CD8 (0.2-0.9) & $0.29(0.02-1.93)$ & $0.20(0.07-0.95)$ & 0.414 \\
\hline CD56 (0.12-0.88) & $0.11(0.00-0.70)$ & $0.15(0.00-0.80)$ & 0.09 \\
\hline $\operatorname{lgG}(6-13 \mathrm{~g} / \mathrm{L})$ & $9.0(2.8-22.6)$ & $8.8(3.0-18.9)$ & 0.823 \\
\hline IgG decline $\geq 30 \%(\%)$ & 20 & 35 & 0.041 \\
\hline Hypogammaglobulinaemia (\%) & 13 & 16 & 0.593 \\
\hline $\operatorname{lgM}(0.4-2.2 \mathrm{~g} / \mathrm{L})$ & $0.7(0.3-2.6)$ & $0.7(0.3-2.0)$ & 0.398 \\
\hline $\lg \mathrm{A}(0.8-3.7 \mathrm{~g} / \mathrm{L})$ & $1.8(0.4-5.3)$ & $2.1(0.5-4.3)$ & 0.715 \\
\hline ANCA-positive (\%) & 73 & 76 & 0.703 \\
\hline \multicolumn{4}{|l|}{ Comorbidities (\%) } \\
\hline COPD & 1 & 6 & 0.053 \\
\hline Diabetes & 6 & 18 & 0.021 \\
\hline Hypertension & 37 & 33 & 0.557 \\
\hline Myocardial infarction/reduced LVEF & 8 & 18 & 0.036 \\
\hline \multicolumn{4}{|l|}{ Indication (\%) } \\
\hline Minor relapse & 41 & 29 & 0.114 \\
\hline Major relapse & 27 & 39 & 0.13 \\
\hline
\end{tabular}


Table 1 Continued

\begin{tabular}{|c|c|c|c|}
\hline & No severe infection $(n=143)$ & Severe infection $(n=49)$ & $P$ values \\
\hline Maintenance & 78 & 82 & 0.622 \\
\hline Refractory disease & 31 & 27 & 0.516 \\
\hline Steroid sparing & 17 & 22 & 0.375 \\
\hline 1st line & 5 & 10 & 0.187 \\
\hline \multicolumn{4}{|l|}{ Premedication (last 12 months) } \\
\hline CYC (g) & $0(0-45)$ & $0(0-22)$ & 0.632 \\
\hline $\operatorname{MMF}(\mathrm{g})$ & $0(0-1080)$ & $15(0-1080)$ & 0.798 \\
\hline AZA (g) & $0(0-81)$ & $0(0-72)$ & 0.036 \\
\hline MTX (mg) & $0(0-1286)$ & $0(0-1286)$ & 0.739 \\
\hline IVIG (ever) (\%) & 4 & 12 & 0.128 \\
\hline Anti-TNF (ever) (\%) & 3 & 5 & 0.65 \\
\hline PLEX (ever) (\%) & 9 & 7 & 1 \\
\hline ALM (ever) (\%) & 5 & 14 & 0.079 \\
\hline \multicolumn{4}{|l|}{ Medication used concurrently with RTX } \\
\hline Steroids (mg) & $15(0-60)$ & $15(5-60)$ & 0.087 \\
\hline Trimethoprim-sulfamethoxazole (\%) & 43 & 22 & 0.009 \\
\hline Other antibiotic prophylaxis (\%) & 9 & 16 & 0.172 \\
\hline
\end{tabular}

Metric variables are shown as median and (minimum-maximum), nominal variables are shown as \%. Statistics tests are $\chi$ quadrate test/Fisher's exact test and Mann-Whitney $U$ test where appropriate. The respective reference ranges, if applicable, are given in parentheses. $P$ values indicating significant changes are highlighted in bold font.

ALM, alemtuzumab; ANCA, antineutrophil cytoplasm antibody; AZA, azathioprine; BVAS, Birmingham Vasculitis Activity Score; CD, cluster of differentiation; CNS, central nervous system; COPD, chronic obstructive pulmonary disease; CRP, C reactive protein; CYC, cyclophosphamide; DEI, Disease Extent Index; eGFR, estimated glomerular filtration rate; EGPA, eosinophilic granulomatosis with polyangiitis; ENT, ear, nose and throat; ESR, erythrocyte sedimentation rate; GPA, granulomatosis with polyangiitis; IVIG, intravenous immunoglobulins; LVEF, left ventricular ejection fraction; MMF, mycophenolate mofetil; MPA, microscopic polyangiitis; MTX, methotrexate; PLEX, plasma exchange; RTX, rituximab; TNF, tumour necrosis factor; WBC, white blood count.

\section{Risk for lower respiratory tract infections after rituximab}

Since respiratory tract infections were the leading cause of infectious complications $(n=63)$, we aimed to identify factors predicting the risk. Nine patients underwent bronchoscopy and most of them had at least two respiratory tract infections (7/9). Patients with preserved eGFR (HR 0.99, 95\% CI 0.98 to 1.00), presenting with sinusitis (HR $0.47,95 \%$ CI 0.23 to 0.98 ) and ENT involvement (HR $0.43,95 \% \mathrm{CI} 0.20$ to 0.87 ) as well as receiving rituximab for refractory disease ( $\mathrm{HR} \quad 0.35,95 \% \mathrm{CI}$ 0.12 to 0.99 ), had a lower likelihood to develop severe pulmonary infections. In contrast, lung involvement (HR 2.53, 95\% CI 1.08 to 5.93 ) and in particular endobronchial involvement (HR $4.30,95 \% \mathrm{CI} 2.06$ to 8.94 ) and severe bronchiectasis (HR 7.48, $95 \%$ CI 2.22 to 25.16 ) emerged as risk factors. Higher CRP (HR 1.01, 95\% CI 1.00 to 1.01), ESR (HR 1.02, 95\% CI 1.00 to 1.03), neutrophils (HR 1.15, 95\% CI 1.15) and WBC (HR 1.07, $95 \%$ CI 1.01 to 1.12 ) at baseline were associated with severe pulmonary infections. Moreover, those with concomitant COPD (HR 19.75, 95\% CI 5.23 to 74.63), major relapse as indication (HR 2.65, 95\% CI 1.28 to 5.49) and higher steroid doses (HR $1.02,95 \%$ CI 1.00 to 1.04 ) had more pulmonary infections. Multivariate analysis retained endobronchial involvement (HR $4.30,95 \%$ CI 2.06 to 8.94), severe bronchiectasis (HR 7.48, 95\% CI 2.22 to 25.16), neutrophil count at baseline (HR 1.19, 95\% CI 1.06 to 1.33 ) and major relapse (HR 2.65, 95\% CI 1.28 to 5.49) as independent risk factors, while rituximab use in the setting of refractory disease was negatively associated with severe pulmonary infections (HR $0.35,95 \% \mathrm{CI} 0.12$ to 0.99 ) (online supplementary table S3).

\section{Prescription pattern and side effects of trimethoprim- sulfamethoxazole}

The dose of trimethoprim-sulfamethoxazole used as a prophylaxis was not consistent. Most patients received $480 \mathrm{mg}$ on alternate days (38.36\%), followed by $960 \mathrm{mg}$ on alternate days
(21.92\%) and $960 \mathrm{mg}$ twice daily $(12.33 \%$, further details see online supplementary table S4). Among differences in the prescription pattern, a diagnosis of GPA, ENT involvement including sinusitis and deafness, mastoiditis and otitis media were associated with a more frequent prescription. Lower CD4 T-cell count as well as cyclophosphamide in the year before and a higher concomitant steroid use led to trimethoprim-sulfamethoxazole prescription (online supplementary table S5). Next, we assessed side effects of trimethoprim-sulfamethoxazole focusing on recently reported adverse events in rheumatological indications. ${ }^{12}$ Trimethoprim-sulfamethoxazole was stopped in five patients due to haematopoietic complications in three (lymphopenia, pancytopenia, neutropenia), sore mouth in one and abnormal liver function test in the remainder. In general, trimethoprim-sulfamethoxazole prophylaxis was maintained for 14.67 months.

\section{DISCUSSION}

Comorbidities, either attributable to active disease or immunosuppression, remain a major issue in the management of AAV. An analysis of the early EUVAS trials revealed that infections contributed to the majority $(28 / 59,48 \%)$ of deaths within the first year of trial inclusion, whereas it is among the three leading causes thereafter $(15 / 74,20 \%)$. A direct effect of induction treatment was proposed to be causative of severe infections within the first year. ${ }^{2}$ A recent study analysing the Chapel Hill cohort highlighted that infections were responsible for a high proportion of deaths within the first year $(4 / 31,13 \%)$, while active disease (29\%) was the leading cause in a large cohort comprising 421 patients with a follow-up of at least 1 year. ${ }^{13}$ Differences in the treatment modalities may have accounted for the differences leading to fatal infections in diverse cohorts. The methylprednisolone versus plasma exchange (MEPEX) trial (one of the early European Vasculitis Society (EUVAS) trials) randomised patients 


\section{Clinical and epidemiological research}

Table 2 Univariate and multivariate analysis of risk factors for severe or life-threatening infection following rituximab treatment during 24 months of follow-up

\begin{tabular}{|c|c|c|c|c|c|c|}
\hline & \multicolumn{3}{|c|}{ Univariate analysis } & \multicolumn{3}{|c|}{ Multivariate analysis } \\
\hline & HR & $95 \% \mathrm{Cl}$ & $P$ values & HR & $95 \% \mathrm{Cl}$ & $P$ values \\
\hline \multicolumn{7}{|l|}{ Demographics } \\
\hline Age (years) & 1.02 & 1.00 to 1.04 & 0.031 & 1.03 & 1.01 to 1.05 & 0.012 \\
\hline Gender (male) & 0.88 & 0.50 to 1.55 & 0.647 & & & \\
\hline \multicolumn{7}{|l|}{ Type of vasculitis } \\
\hline GPA & Reference & - & - & & & \\
\hline MPA & 1.59 & 0.78 to 3.23 & 0.203 & & & \\
\hline EGPA & 0.95 & 0.42 to 2.15 & 0.899 & & & \\
\hline \multicolumn{7}{|l|}{ Symptoms/manifestations } \\
\hline B-symptoms (night sweat, fever, unintentional weight loss) & 0.67 & 0.28 to 1.58 & 0.355 & & & \\
\hline Neuropathy & 0.89 & 0.46 to 1.70 & 0.72 & & & \\
\hline Sinusitis & 0.48 & 0.27 to 0.84 & 0.01 & & & \\
\hline Deafness/mastoiditis/otitis media & 0.69 & 0.35 to 1.35 & 0.275 & & & \\
\hline Arthralgia & 0.63 & 0.35 to 1.14 & 0.127 & & & \\
\hline \multicolumn{7}{|l|}{ Organ involvement } \\
\hline CNS & 1.12 & 0.35 to 3.63 & 0.85 & & & \\
\hline Subglottic/tracheal stenosis & 1.14 & 0.51 to 2.54 & 0.746 & & & \\
\hline Skin & 0.74 & 0.33 to 1.64 & 0.45 & & & \\
\hline Kidney & 1.27 & 0.72 to 2.21 & 0.411 & & & \\
\hline Eye & 0.52 & 0.23 to 1.17 & 0.113 & & & \\
\hline Others & 0.82 & 0.26 to 2.65 & 0.745 & & & \\
\hline ENT & 0.46 & 0.26 to 0.82 & 0.008 & & & \\
\hline Lung & 1.57 & 0.87 to 2.82 & 0.136 & & & \\
\hline \multicolumn{7}{|l|}{ Imaging findings } \\
\hline Pulmonary cavities & 1.11 & 0.56 to 2.21 & 0.765 & & & \\
\hline Endobronchial & 2.44 & 1.38 to 4.32 & 0.002 & 2.21 & 1.14 to 4.26 & 0.018 \\
\hline Severe bronchiectasis & 4.79 & 1.47 to 15.59 & 0.009 & & & \\
\hline \multicolumn{7}{|l|}{ Disease activity measures } \\
\hline BVAS & 1.01 & 0.95 to 1.07 & 0.811 & & & \\
\hline DEI & 0.98 & 0.84 to 1.15 & 0.840 & & & \\
\hline \multicolumn{7}{|l|}{ Laboratory values } \\
\hline Creatinine & 1 & 1.00 to 1.00 & 0.141 & & & \\
\hline eGFR (MDRD equation) $\mathrm{mL} / \mathrm{min} / 1.73 \mathrm{~m}^{2}$ & 0.99 & 0.98 to 1.00 & 0.011 & & & \\
\hline CRP & 1.01 & 1.00 to 1.01 & 0.061 & & & \\
\hline ESR & 1.01 & 1.00 to 1.02 & 0.014 & & & \\
\hline Neutrophils & 1.11 & 1.03 to 1.20 & 0.005 & & & \\
\hline WBC & 1.06 & 1.01 to 1.10 & 0.013 & & & \\
\hline Lymphocytes & 0.73 & 0.48 to 1.11 & 0.142 & & & \\
\hline CD19 & 1.17 & 0.11 to 12.51 & 0.896 & & & \\
\hline CD3 & 0.75 & 0.45 to 1.25 & 0.27 & & & \\
\hline CD4 & 0.74 & 0.34 to 1.61 & 0.44 & & & \\
\hline CD8 & 0.58 & 0.16 to 2.09 & 0.407 & & & \\
\hline CD56 & 2.75 & 0.34 to 22.10 & 0.341 & & & \\
\hline $\lg G$ & 1.02 & 0.93 to 1.12 & 0.663 & & & \\
\hline $\lg$ decline $\geq 30 \%$ & 1.88 & 1.04 to 3.39 & 0.036 & & & \\
\hline Hypogammaglobulinaemia & 1.22 & 0.54 to 2.74 & 0.633 & & & \\
\hline $\lg M$ & 0.72 & 0.38 to 1.35 & 0.304 & & & \\
\hline $\lg A$ & 1.11 & 0.80 to 1.54 & 0.535 & & & \\
\hline ANCA positive & 1.11 & 0.58 to 2.14 & 0.744 & & & \\
\hline \multicolumn{7}{|l|}{ Comorbidities } \\
\hline COPD & 16.07 & 4.41 to 58.49 & $<0.001$ & 6.3 & 1.08 to 36.75 & 0.041 \\
\hline Diabetes & 2.35 & 1.14 to 4.85 & 0.021 & & & \\
\hline Hypertension & 0.79 & 0.44 to 1.44 & 0.445 & & & \\
\hline Myocardial infarction/reduced LVEF & 2.21 & 1.07 to 4.56 & 0.032 & & & \\
\hline
\end{tabular}


Table 2 Continued

\begin{tabular}{|c|c|c|c|c|c|c|}
\hline & \multicolumn{3}{|c|}{ Univariate analysis } & \multicolumn{3}{|c|}{ Multivariate analysis } \\
\hline & HR & $95 \% \mathrm{Cl}$ & $P$ values & HR & $95 \% \mathrm{Cl}$ & $P$ values \\
\hline Minor relapse & 0.6 & 0.32 to 1.11 & 0.102 & & & \\
\hline Maintenance & 1.15 & 0.56 to 2.37 & 0.708 & & & \\
\hline Refractory disease & 0.8 & 0.42 to 1.51 & 0.491 & & & \\
\hline Steroid sparing & 1.37 & 0.70 to 2.68 & 0.36 & & & \\
\hline CYC (g) & 0.97 & 0.90 to 1.04 & 0.389 & & & \\
\hline $\operatorname{MMF}(\mathrm{g})$ & 1 & 1.00 to 1.00 & 0.273 & & & \\
\hline AZA (g) & 0.97 & 0.95 to 1.00 & 0.066 & & & \\
\hline MTX (mg) & 1 & 1.00 to 1.00 & 0.979 & & & \\
\hline IVIG (ever) & 2.4 & 0.94 to 6.12 & 0.067 & & & \\
\hline \multicolumn{7}{|l|}{ Medication used concurrently with RTX } \\
\hline Steroids (mg) & 1.02 & 1.01 to 1.04 & 0.006 & & & \\
\hline Trimethoprim-sulfamethoxazole & 0.45 & 0.23 to 0.88 & 0.02 & 0.3 & 0.13 to 0.69 & 0.005 \\
\hline Other antibiotic prophylaxis & 1.63 & 0.76 to 3.47 & 0.209 & & & \\
\hline
\end{tabular}

Demographics of the respective patients, the form of ANCA-associated vasculitis, symptoms, laboratory values, comorbidities, indication for rituximab use, the premedication and the concomitant therapy are given. $P$ values indicating significant changes are highlighted in bold font.

ALM, alemtuzumab; ANCA, antineutrophil cytoplasm antibody; AZA, azathioprine; BVAS, Birmingham Vasculitis Activity Score; CD, cluster of differentiation; CNS, central nervous system; COPD, chronic obstructive pulmonary disease; CRP, C reactive protein; CYC, cyclophosphamide; DEI, Disease Extent Index; eGFR, estimated glomerular filtration rate; EGPA, eosinophilic granulomatosis with polyangiitis; ENT, ear, nose and throat; ESR, erythrocyte sedimentation rate; GPA, granulomatosis with polyangiitis; IVIG, intravenous immunoglobulins; LVEF, left ventricular ejection fraction; MMF, mycophenolate mofetil; MPA, microscopic polyangiitis; MTX, methotrexate; PLEX, plasma exchange; RTX, rituximab; TNF, tumour necrosis factor; WBC, white blood count.

either to plasma exchange or high-dose methylprednisolone alongside standard induction therapy reported 19 deaths (out of 137 patients) related to infections within the first year. ${ }^{14}$

Little is known about infections in patients with AAV treated with rituximab. In the first 6 months, the rate of severe infections (defined as grade $\geq 3$ CTCAE V.3.0 event) was $7 \%$ in the group of patients receiving either rituximab or standard of care in the RAVE trial. ${ }^{4}$ Over 18 months, $12 \%$ in the rituximab and $11 \%$ of participants in the standard of care group had at least one

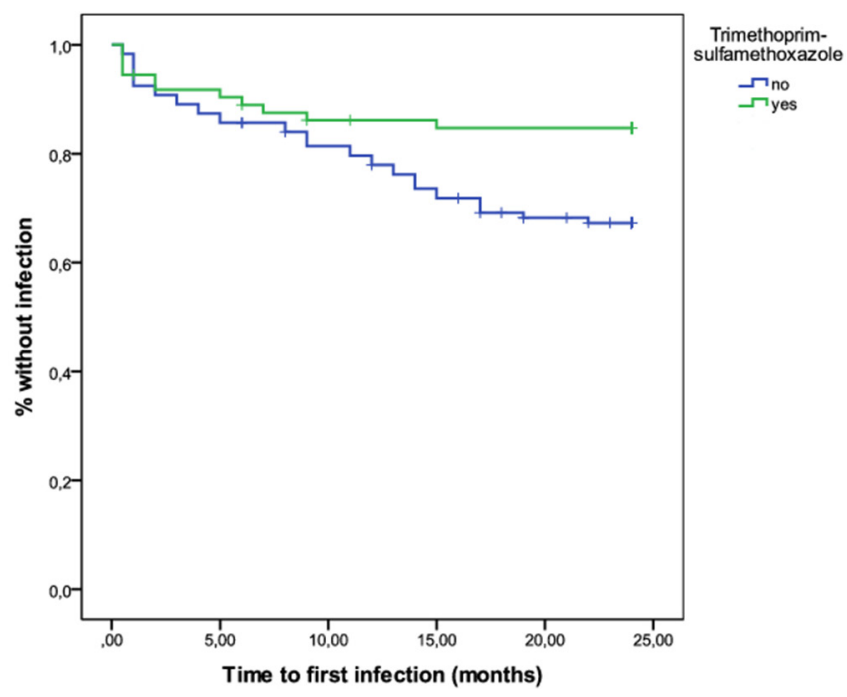

Figure 1 Kaplan-Meier curve of patients presenting with severe infections and either receiving trimethoprim-sulfamethoxazole or prophylaxis or not. episode of grade $\geq 3$ infections. ${ }^{6}$ In the RITUXVAS trial, a higher occurrence of severe infectious complications was observed in both treatment arms. While the rate of severe infections was $18 \%$ in both arms, the number of patients presenting with non-severe infections was higher (18\% vs 9\%) in the rituximab group. ${ }^{5}$ In general, patients with vasculitis may carry an increased risk to develop severe infections following rituximab administration. In patients with rheumatoid arthritis (RA), long-term follow-up of a global clinical trial programme revealed a serious infection event rate of 3.76 per 100 person-years. In contrast to our findings, opportunistic infections remained rare during follow-up with an event rate of 0.05 events per 100 patient-years in the RA cohort. ${ }^{15}$

The current European League Against Rheumatism/European Renal Association - European Dialysis and Transplant Association (EULAR/ERA-EDTA) recommendations for the management of AAV encourage P. jirovecii prophylaxis in patients receiving cyclophosphamide. ${ }^{16}$ However, no concrete recommendation concerning rituximab is given. In the updated EMA label, prophylaxis is recommended during and following rituximab, as appropriate. ${ }^{17}$ In this study, the frequency of $P$. jirovecii infection was low $(\mathrm{n}=1)$. This frequency is in line with a study reporting one case of $P$. jirovecii in patients receiving mainly cyclophosphamide as induction treatment. ${ }^{13}$ Currently, it is uncertain if patients with AAV receiving rituximab benefit from $P$. jirovecii prophylaxis since the reported frequency of severe adverse events attributable to trimethoprim-sulfamethoxazole is high in patients with systemic autoimmune diseases, with some fatalities. ${ }^{12} 18 \mathrm{~A}$ randomised controlled trial investigating the role of trimethoprim-sulfamethoxazole in therapeutic dosage (960 $\mathrm{mg}$ twice a day for 2 years) found a reduction in respiratory 
tract infections and a trend towards fewer non-respiratory tract infections $(p=0.05)$ compared with placebo. ${ }^{19}$ This is in line with our study confirming a protective effect of prophylactic trimethoprim-sulfamethoxazole use on the risk to develop severe infections. Thus, it may be appropriate to conclude that trimethoprim-sulfamethoxazole may reduce $P$. jirovecii pneumonia and also reduces overall infective risk and prophylaxis should be initiated in patients with AAV receiving rituximab. In our cohort, patients tolerating trimethoprim-sulfamethoxazole remained on prophylaxis during the 2 -year period (mean 14.67 months). Five patients stopped trimethoprim-sulfamethoxazole due to adverse events.

The reported occurrence of severe infections in observational studies of AAV varies (frequency 20\%-60\%) 3 influenced by follow-up times, prophylactic measures and the impact of different criteria for infections. In our study, $26.06 \%$ patients presented with at least one severe infection. The observed frequency is higher compared with both Rituximab versus Cyclophosphamide for ANCA-Associated Vasculitis (RAVE) and Rituximab versus Cyclophosphamide in ANCA-Associated Renal Vasculitis (RITUXVAS) trials. This may be explained by the scheduled rigorous study visits, allowing for early detection of infection and prescription of antimicrobials, or the selection of a lower risk cohort for the clinical trials. However, the frequency of observed severe infections is similar to other observational studies reported to date. ${ }^{720}$ Older age was an independent risk factor for infections in the pre-rituximab era. ${ }^{3}$ We observed an association between age and severe infections in our cohort. Patients with lung involvement and concomitant COPD may be particularly vulnerable to severe infections. Endobronchial involvement and COPD were risk factors for infections and endobronchial involvement alongside severe bronchiectasis predictors of severe pulmonary infections. In patients with AAV on immunosuppressive treatment, most severe infections are located in the respiratory tract. ${ }^{132122}$ In rituximab-treated patients, 20 out of 30 infectious complications were restricted to the upper and lower respiratory tract during a follow-up period of 230.4 patient-years. ${ }^{8}$ Respiratory tract infections were the leading cause of severe infections in our cohort as well.

Compared with a matched background population, patients with AAV are at an increased risk of severe infections, including non-specific (HR 4.55), Gram-negative (HR 3.49) and $S$. aureus septicaemia (HR 3.40), pneumonia (HR 3.27), acute upper respiratory tract infections (HR 8.88), C. difficile infection (HR 5.35) and skin infections (HR 5.35). ${ }^{23}$ Interestingly, no difference related to infectious complications was observed when an early cohort was compared with a recent cohort. ${ }^{23}$ Another study corroborated an impact of $S$. aureus in patients with AAV, being the most prevalent causative organism (34\% of 249 positive cultures). Among 85 positive cultures, 18 (21\%) of $S$. aureus isolates were grown despite trimethoprim-sulfamethoxazole prophylaxis. Moreover, 14\% of infections caused by S. aureus were severe. ${ }^{13}$ In contrast, our study found a broad spectrum of opportunistic pathogens and P. aeruginosa as well as $S$. aureus (four severe infections, each) were the leading causative organism, followed by E. coli (three severe infections). The spectrum of isolates is in line with a recent study reporting the efficacy and safety profile of rituximab in induction and maintenance of remission. Out of 12 severe infections, four led to fatality in four subjects with either coma (meningitis) or respiratory failure (pneumonitis with detection of $P$. aeruginosa or $P$. jirovecii). ${ }^{7}$ Both $S$. aureus and Gram-negative bacteria may have a direct impact on disease onset or relapse, ${ }^{24}$ which is a potential explanation for the high number of infections caused by these pathogens.

Most infections occur within the first months of treatment. McGregor et al showed the highest risk of infections during the first 3 months of follow-up and in general severe infections within the first 12 months were associated with death (19\% vs 4\%). ${ }^{13}$ A recent registry analysis highlighted that a high proportion of severe infections occurred during the first 6 months of follow-up $(38.4 \%) .^{23}$ In contrast, severe infections occurred during the whole observational period in our cohort of rituximab-treated patients. In retrospective studies, hypogammaglobulinaemia was a frequently observed complication of rituximab with the need of IgG replacement due to recurrent infections in $4.2 \%$ of the patients. ${ }^{25}$ Univariate analysis revealed an association between IgG decline of at least 30\% from baseline in patients with severe infections. This may indicate that this subgroup of patients with a drop in IgG levels may be specifically prone towards infections.

In conclusion, we found severe infections occurring in approximately one quarter of patients in a 2-year observation period after rituximab therapy for AAV. There was a reduction of severe infections when trimethoprim-sulfamethoxazole prophylaxis was used. Respiratory tract infections were the leading cause of severe infections. We found an association of endobronchial involvement, bronchiectasis and rituximab use for major relapses with severe respiratory tract infections. While these results require confirmation, they support routine use of trimethoprim-sulfamethoxazole in rituximab-treated patients.

Contributors AK, SG and JT retrieved data. Analysis was performed by AK, JK and SG. All authors contributed to the analysis or interpretation of data and critical revision of the manuscript.

Funding The authors have not declared a specific grant for this research from any funding agency in the public, commercial or not-for-profit sectors.

Disclaimer The funding bodies did not have any contribution in collection, analysis, interpretation of data presented or writing of the manuscript.

Competing interests AK has received travel support from Roche/Genentech. DRWJ has received research grants and consulting fees from Roche/Genentech and Terumo BCT and is supported by the Cambridge Biomedical Research Centre.

Patient consent Not required.

Ethics approval Both universities waive specific IRB approval for retrospective analyses.

Provenance and peer review Not commissioned; externally peer reviewed.

Open access This is an open access article distributed in accordance with the Creative Commons Attribution Non Commercial (CC BY-NC 4.0) license, which permits others to distribute, remix, adapt, build upon this work non-commercially, and license their derivative works on different terms, provided the original work is properly cited, appropriate credit is given, any changes made indicated, and the use is non-commercial. See: http://creativecommons.org/licenses/by-nc/4.0/.

(C) Article author(s) (or their employer(s) unless otherwise stated in the text of the article) 2018. All rights reserved. No commercial use is permitted unless otherwise expressly granted.

\section{REFERENCES}

1 Jayne D, Rasmussen N. Twenty-five years of European Union collaboration in ANCAassociated vasculitis research. Nephrol Dial Transplant 2015;30(Suppl 1):i1-7.

2 Flossmann 0, Berden A, de Groot K, et al. Long-term patient survival in ANCAassociated vasculitis. Ann Rheum Dis 2011;70:488-94.

3 Kronbichler A, Jayne DR, Mayer G. Frequency, risk factors and prophylaxis of infection in ANCA-associated vasculitis. Eur J Clin Invest 2015;45:346-68.

4 Stone JH, Merkel PA, Spiera R, et al. Rituximab versus cyclophosphamide for ANCAassociated vasculitis. N Engl J Med 2010;363:221-32

5 Jones RB, Tervaert JW, Hauser T, et al. Rituximab versus cyclophosphamide in ANCAassociated renal vasculitis. N Eng/ J Med 2010;363:211-20.

6 Specks U, Merkel PA, Seo P, et al. Efficacy of remission-induction regimens for ANCAassociated vasculitis. N Engl J Med 2013;369:417-27.

7 Charles P, Néel A, Tieulié N, et al. Rituximab for induction and maintenance treatment of ANCA-associated vasculitides: a multicentre retrospective study on 80 patients. Rheumatology 2014;53:532-9. 
8 Cartin-Ceba R, Golbin JM, Keogh KA, et al. Rituximab for remission induction and maintenance in refractory granulomatosis with polyangiitis (Wegener's): ten-year experience at a single center. Arthritis Rheum 2012;64:3770-8.

9 Brunetta P, Martin C, Lehane PB, et al. Two Case Reports of Progressive Multifocal Leukoencephalopathy (PML) in Granulomatosis Polyangiitis (GPA) Treated With Rituximab. 17th International ANCA and Vasculitis Workshop 2015. London, United Kingdom.

10 Watts R, Lane S, Hanslik T, et al. Development and validation of a consensus methodology for the classification of the ANCA-associated vasculitides and polyarteritis nodosa for epidemiological studies. Ann Rheum Dis 2007;66:222-7.

11 Institute NC. Common Terminology Criteria for Adverse Events (CTCAE) Version 4.0 2009 https://evs.nci.nih.gov/ftp1/CTCAE/CTCAE_4.03_2010-06-14_QuickReference_ 5x7.pdf.

12 Park JW, Curtis JR, Moon J, et al. Prophylactic effect of trimethoprimsulfamethoxazole for Pneumocystis pneumonia in patients with rheumatic diseases exposed to prolonged high-dose glucocorticoids. Ann Rheum Dis 2018;77:644-9.

13 McGregor JG, Negrete-Lopez R, Poulton CJ, et al. Adverse events and infectious burden, microbes and temporal outline from immunosuppressive therapy in antineutrophil cytoplasmic antibody-associated vasculitis with native renal function. Nephrol Dial Transplant 2015;30(Suppl 1):i171-81.

14 Jayne DR, Gaskin G, Rasmussen N, et al. Randomized trial of plasma exchange or high-dosage methylprednisolone as adjunctive therapy for severe renal vasculitis. J Am Soc Nephrol 2007;18:2180-8.

15 van Vollenhoven RF, Fleischmann RM, Furst DE, et al. Longterm safety of rituximab: final report of the Rheumatoid Arthritis Global Clinical Trial Program over 11 years. J Rheumatol 2015;42:1761-6.

16 Yates M, Watts RA, Bajema IM, et al. EULAR/ERA-EDTA recommendations for the management of ANCA-associated vasculitis. Ann Rheum Dis 2016;75:1583-94.
17 Summary of product characteristics rituximab. $2017 \mathrm{http}: / /$ www.ema.europa.eu/ docs/en_GB/document_library/EPAR_-_Product_Information/human/000165/ WC500025821.pdf

18 Utsunomiya M, Dobashi H, Odani T, et al. Optimal regimens of sulfamethoxazoletrimethoprim for chemoprophylaxis of Pneumocystis pneumonia in patients with systemic rheumatic diseases: results from a non-blinded, randomized controlled trial. Arthritis Res Ther 2017;19:7.

19 Stegeman CA, Tervaert JW, de Jong PE, et al. Trimethoprim-sulfamethoxazole (co-trimoxazole) for the prevention of relapses of Wegener's granulomatosis. Dutch Co-Trimoxazole Wegener Study Group. N Engl J Med 1996;335:16-20.

20 Calich AL, Puéchal X, Pugnet G, et al. Rituximab for induction and maintenance therapy in granulomatosis with polyangiitis (Wegener's). Results of a single-center cohort study on 66 patients. J Autoimmun 2014;50:135-41.

21 Goupil R, Brachemi S, Nadeau-Fredette AC, et al. Lymphopenia and treatment-related infectious complications in ANCA-associated vasculitis. Clin J Am Soc Nephrol 2013;8:416-23.

22 Harper L, Savage CO. ANCA-associated renal vasculitis at the end of the twentieth century - a disease of older patients. Rheumatology 2005;44:495-501.

23 Mohammad AJ, Segelmark M, Smith R, et al. Severe infection in antineutrophil cytoplasmic antibody-associated vasculitis. J Rheumatol 2017;44:1468-75.

24 Kronbichler A, Kerschbaum J, Mayer G. The influence and role of microbial factors in autoimmune kidney diseases: a systematic review. J Immunol Res 2015;2015:1-13.

25 Roberts DM, Jones RB, Smith RM, et al. Rituximab-associated hypogammaglobulinemia: incidence, predictors and outcomes in patients with multisystem autoimmune disease. J Autoimmun 2015;57:60-5. 